

\title{
RESEÑA: Foro de revisión y análisis de la iniciativa de Ley General de Biodiversidad como contribución de BCS a la defensa de la biodiversidad en México
}

\author{
Jesús Echevarría-Haro*, María de La Paz Gálvez-Ballesteros, \\ Miguel Ángel Leal-Jiménez, José Luis López-López, \\ María Concepción Lora-Vilchis y Janette Murillo-Jiménez
}

El pasado 25 de octubre de 2016, se presentó en la Cámara de Senadores, una iniciativa con proyecto de decreto por el que se expide la Ley General de Biodiversidad (LGB). La iniciativa fue presentada por la Senadora Ninfa Salinas Sada, del Partido Verde Ecologista de México (PVEM). En la exposición de motivos se indica que la iniciativa tiene por objeto integrar en un solo ordenamiento las disposiciones transversales y señala la concurrencia entre los tres niveles de gobierno, absorbe lo previsto en el Título Segundo de la LGEEPA, y se argumenta que se fortalecerán y actualizarán las disposiciones de Ley General de Vida Silvestre (LGVS), que se abrogaría esta ley; se indica también que incluye diversas disposiciones del Protocolo de Nagoya sobre el acceso a recursos genéticos, y señala que tiene el afán de conservar la biodiversidad de México.

Lo anterior ha generado diversas manifestaciones de inconformidad y protesta a nivel nacional, una de ellas dio origen a la integración de un COLECTIVO ACADÉMICO SUDCALIFORNIANO constituido por académicos de diversas instituciones de Baja California Sur. Este colectivo, preocupado por las posibles afectaciones a la biodiversidad en general en México, pero en especial en Baja California Sur, convocó a investigadores, maestros y estudiantes de instituciones de investigación y educación superior, así como a representantes de gobierno, organizaciones no gubernamentales y de la sociedad civil, a participar en un FORO para revisar y analizar la iniciativa de la Ley General de Biodiversidad.

\footnotetext{
* Autor corresponsal: jeh944@hotmail.com
} 
El Foro se realizó el 16 de junio en el Centro Cultural La Paz con la asistencia de 94 personas; inició con la inauguración por el Dr. Alfredo Ortega Rubio del CIBNOR quien explicó brevemente algunos de los puntos más inquietantes de la iniciativa. Acto seguido se presentaron tres videoconferencias, en las que participaron el M. en C. Roberto de la Maza Hernández, de CEIBA, y los doctores Luis Fueyo Mac Donald Coordinador de Red Mexicana - Agenda 2030 y ODS de la ONU, y Exequiel Escurra Real de Azua, Director del Programa US MEXUS, quienes presentaron su visión de la iniciativa y de sus afectaciones.

El Foro continuó con la revisión y análisis de la iniciativa, a través de 4 temas principales y se discutió por mesas de trabajo: Mesa 1) Disposiciones Generales, Participación Social e Información sobre la Biodiversidad; Mesa 2) Diversidad Genética y apoyo a esfuerzos para la Conservación de la Vida Silvestre; Mesa 3) Diversidad de Especies; Mesa 4) Diversidad de Ecosistemas.

A continuación, se describen algunas de las observaciones hechas por los participantes en las diferentes mesas:

1.- La iniciativa se presentó en forma muy apresurada y poco revisada, incluso tiene transcripciones textuales de la LGVS que se salen de contexto por ejemplo "colecta científica y aprovechamiento extractivo". Por otra parte, en el artículo 110, se menciona algo considerado como un absurdo, como es el que cuando una especie desaparezca de su ambiente natural podrá ser sustituida por una subespecie genéticamente similar, lo cual no tiene ningún fundamento crítico y es contrario a la conservación.

2.- Es evidente que esta iniciativa, no ha sido sometida a una amplia consulta por expertos, carece de un glosario de los términos citados en varios artículos, y no hace referencia a cuáles son las facultades de cada una de las Leyes Específicas, como la Ley General de Desarrollo Forestal Sustentable, LGVS y LGEEPA. Por lo que, de ser aprobada esta iniciativa, estaría debilitando la legislación actual, ya que diluiría las facultades legislativas o las eliminaría (LGVS).

Entre los participantes predominó la idea de que en lugar de crear una LGB, se debe revisar y complementar la LGEEPA y la LGVS, de manera amplia, responsable e incluyente, por los diversos sectores de la sociedad, para adecuar el marco jurídico que incluya el tema de la biodiversidad de manera seria y actualizada para que la nación conserve la patria potestad y proteja nuestra riqueza natural y el bien común. 
3.- Al abrogar la LGVS y no especificar el manejo de las zonas de manglares, la iniciativa deja fuera los Convenios Internacionales que México ha suscrito (RAMSAR), lo cual afectaría el cumplimiento de la política internacional de conservación y sustentabilidad. Esta omisión flexibiliza la intervención de proyectos productivos de alto impacto socioecológico en las zonas costeras, desprotegiendo la biodiversidad y poniendo en riego los servicios ambientales de estos ecosistemas costeros.

4.- Con relación a las áreas naturales protegidas (ANP), esta iniciativa facilita la intervención, desdibujando el papel normativo de los Programas de Manejo y obviando la construcción de instrumentos de protección de los Espacios Prioritarios de Conservación (EPC).

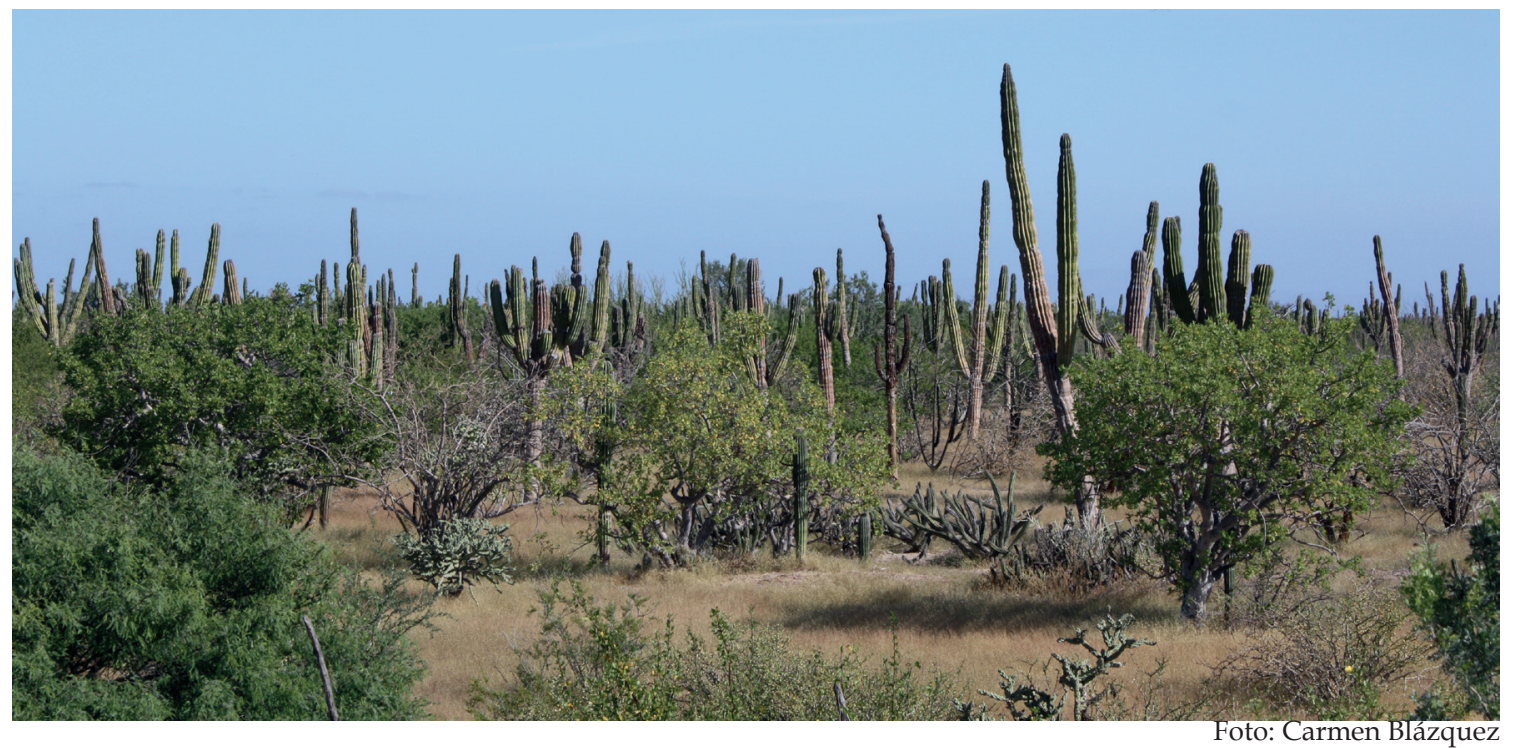

5.- Se rechaza en forma categórica la apropiación de los derechos de decisión sobre la biodiversidad a una sola instancia, toda vez que debe ser un proceso que involucre a todos los sectores de la sociedad, con fundamento en los principios básicos de la democracia y en las disposiciones constitucionales. Por lo anterior, es importante incluir en la consulta pública y el consentimiento previo, libre e informado a las comunidades originarias considerando los mecanismos propios de estas comunidades y sus tradiciones culturales. La participación de estos grupos y sus conocimientos tradicionales en el uso manejo y preservación de su biodiversidad (flora y fauna) deben ser respetados ya que son propiedad de esas comunidades y consideramos que su participación es fundamental.

6.- Se identifica como una seria inconstitucionalidad al derecho de participación social e 
informada de la cuál es garante nuestra constitución, pues en el artículo $6^{\circ}$ transitorio de esta iniciativa en comento, se emite que, en un plazo no mayor de 300 días naturales a partir de la publicación del Decreto, todos los decretos correspondientes a las ANP deberán regularizarse en conformidad con lo que establece esta iniciativa de Ley. Esto atenta contra los procesos de participación social e incluyente requeridos para el establecimiento y la implementación de los Programas de Manejo de las ANP que en general requieren de varios años.

7.- Algo muy evidente es que existen serias deficiencias respecto al conocimiento de nuestra biodiversidad, tanto a nivel de especie como de subespecie y sobre todo a nivel de genoma, donde el problema es mucho mayor. Esto plantea el problema de cómo proteger la biodiversidad, parte de la cual aún desconocemos. Lo anterior coloca a nuestro país en una seria posición de vulnerabilidad con relación a lo referente a propiedad intelectual en relación al Protocolo de Nagoya. Para poder lograr esta protección y regular su manejo es urgente conocerla y documentarla, y difícilmente CONABIO con su actual presupuesto y condiciones podrá enfrentar este enorme reto. Por ello, se requiere de crear una institución especializada que involucre ciencia y tecnología, con presupuesto propio, que evalúe la Diversidad Genética e incluya especies endémicas o de distribución restringida, así como

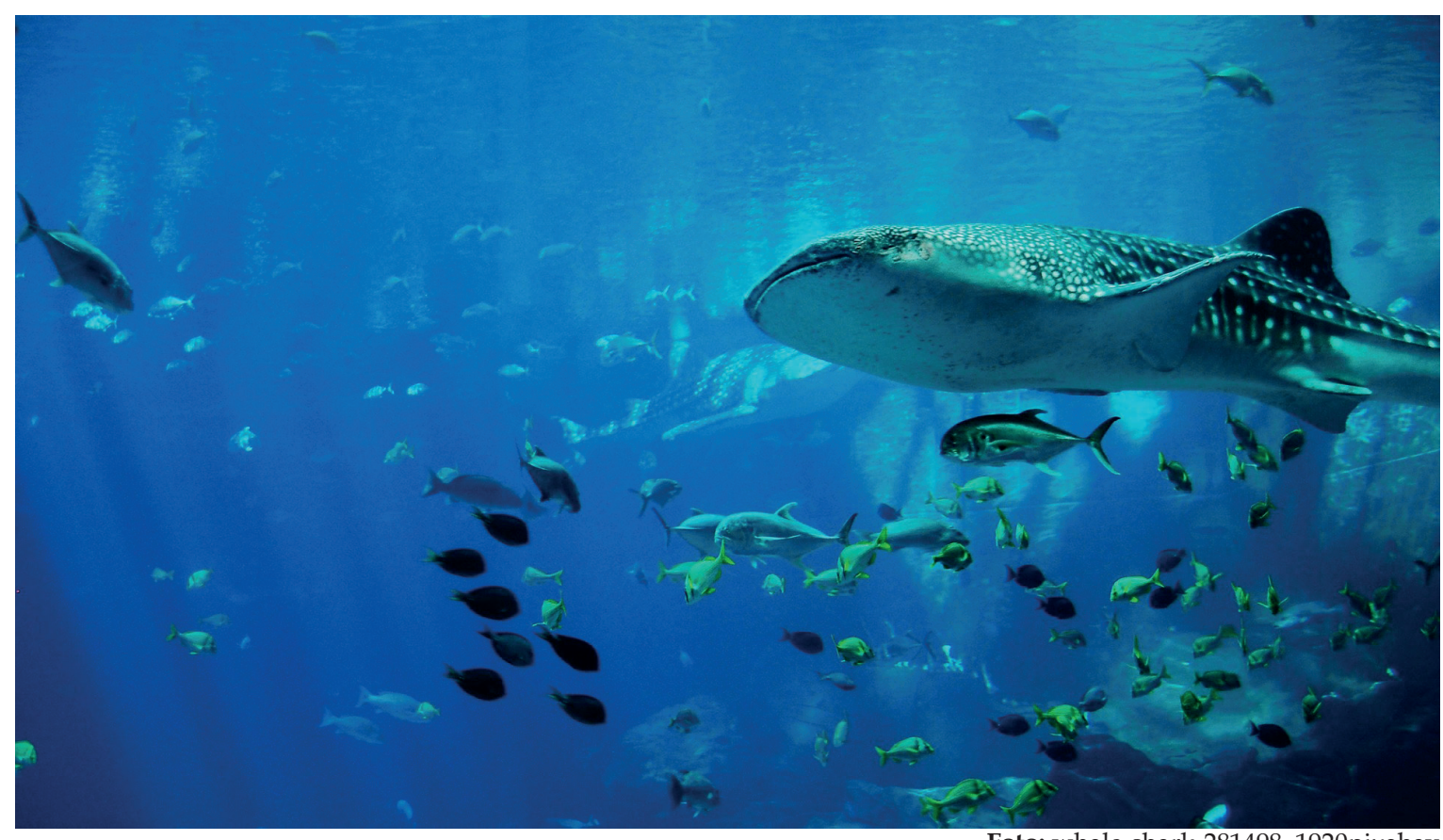

Foto: whale-shark-281498_1920pixabay 
especies marinas y costeras, tanto de flora como de fauna.

8.- En esta propuesta y en toda la legislación ambiental nacional y en el marco institucional ambiental, hay una gran omisión y falta de normatividad referente a la biodiversidad de ecosistemas marinos, costeros y cuerpos de agua.

9.- Es urgente que el país emprenda iniciativas de política pública para el conocimiento de la biodiversidad en los ecosistemas y que en la creación de nuevas ANPs; es fundamental que se asegure la conectividad de los ecosistemas y así garantizar la existencia de especies, acotar polígonos a nivel Nacional, en Zonas Geográficas, además de reforzar el monitoreo

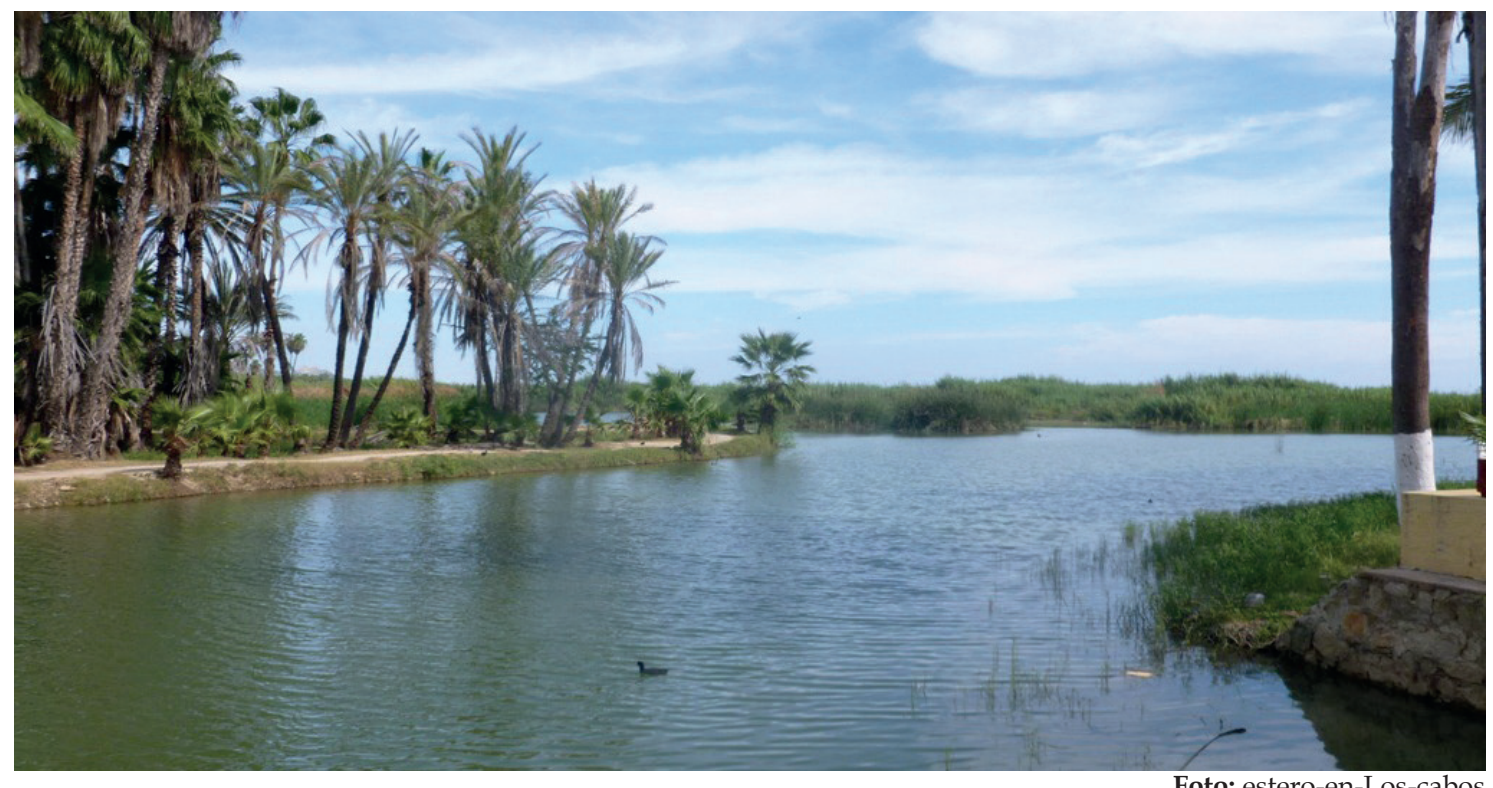

Foto: estero-en-Los-cabos

constante y programas de vigilancia en las ANPs.

10.- Debido a la Falta de COORDINACION DE SECRETARÍAS Y DEPENDENCIAS GUBERNAMENTALES, se propone la creación, en los ámbitos estatal y nacional, de un Consejo Asesor integrado por CONANP, CONABIO, CONACYT, Centros de Investigación y Académicos, para emitir un diagnóstico para el manejo de especies en riesgo para su distribución, reincorporación o repoblación de especies amenazadas en peligro de extinción y manejo integral, que pueda influir en la toma de Decisiones que lleve a cabo la SEMARNAT, mediante la opinión técnica del Consejo.

11.- En todos los puntos donde se faculta a la Secretaría para establecer EPC, deberá consultarse a la sociedad, a los especialistas y a los Consejos, para que no sea una facultad única y 
prerrogativa de la Secretaría, ya que se observa un alto riesgo la regulación normativa de la biodiversidad por parte de la Secretaría, al sobre facultarla otorgando total poder de decisión en la toma de decisiones del manejo y diversidad de ecosistemas.

12.- Una importante omisión de esta iniciativa es lo referente a la flora pues esta se carga más bien hacia la fauna, sobre todo a la terrestre, debiendo incluirse a todas las especies de vida silvestre, incluyendo las marinas y debiendo también considerar de alguna forma a la microbiota, tanto terrestre como marina.

13.- Es importante reforzar el Monitoreo Constante y Programas de Vigilancia en las ANPs.

14.- Una conclusión general del Foro es que, si bien una forma de garantizar la conservación de algunas especies es mediante un aprovechamiento sustentable de la misma, preocupa que en la iniciativa se le dé mucha mayor importancia al aprovechamiento mercantil, que a la propia estabilidad de la biodiversidad y por ende de los ecosistemas.

El foro de consulta terminó a las 15:40 h, quedando tareas por concluir de las cuatro mesas de trabajo, para presentar el documento final, con las conclusiones y propuestas, para entregarse a la brevedad posible al Congreso de la Unión.

El documento será difundido a través de redes sociales y vínculos o enlaces de instituciones de investigación y educación superior.

\section{Agradecimientos}

Los Autores agradecemos a los ponentes y participantes quienes contribuyeron con sus valiosas observaciones y al Lic. Gerardo Hernández el diseño gráfico editorial

\section{Cita}

Echevarría-Haro J.*, M.P. Gálvez-Ballesteros, M. A. Leal-Jiménez, J. L. López-López, M. C. LoraVilchis y J. Murillo-Jiménez. 2017. Reseña del Foro de revisión y análisis de la iniciativa de ley general de biodiversidad como contribución de BCS a la defensa de la biodiversidad en México. Áreas Naturales Protegidas Scripta, 2017. Vol. 3 (1): 59-64. https://doi.org/ 10.18242/anpscripta.2017.03.03.01.0003 